\title{
Primary Aldosteronism with Bilateral Multiple Aldosterone-Producing Adrenal Adenomas
}

\author{
Akira Matsuda, Mutsuo Beniko, Akemi Ikota, Masao Yamazaki, Shigeki Koizumi, Hiroaki Mizumoto, \\ Takeo Watanabe, Kumiko Matsuya, Haruhiko Kunita, Yasuo Mashio and Hironobu Sasano*
}

\begin{abstract}
A 41-year-old woman developed primary aldosteronism due to bilateral multiple aldosteroneproducing adenomas (APA). She was suspected to have idiopathic hyperaldosteronism (IHA) 7 years previously. Although preoperative data suggested APA and IHA was suspected in a postoperative microscopic specimen, a definite clinical diagnosis could not be made. Cytochrome P-450 and other enzymes involved in aldosterone synthesis were found in the tumor portions but not in the zona glomerulosa of attached adrenals, which histopathologically showed "paradoxical hyperplasia". This was a rare case of bilateral multiple APA, which could be differentiated from IHA by immunohistochemical analysis of adrenal steroidogenic enzymes.
\end{abstract}

(Internal Medicine 35: 970-975, 1996)

Key words: aldosterone-producing adenoma, bilateral adrenocortical lesions, steroidogenic enzymes

\section{Introduction}

Primary aldosteronism is usually caused by an adenoma or hyperplasia of the glomerulosa cells in the adrenal cortex. Primary aldosteronism with bilateral adrenal lesions is usually diagnosed with idiopathic hyperaldosteronism (IHA). However, primary aldosteronism with bilateral multiple aldosterone-producing adenomas (APA) is very rare (1). It is difficult to differentiate APA from IHA by the usual endocrinological and histopathological examinations. We report here a case of bilateral multiple APA which could be differentiated from IHA by immunohistochemical analysis of adrenal steroidogenic enzymes.

For editorial comment, see p 919.

\section{Materials and Methods}

Endocrine examinations: Plasma renin activity (PRA) and plasma aldosterone concentration (PAC) were measured by RIA. Urine 17-hydroxycorticosteroids (17-OHCS) and 17keto-steroids (17-KS) were measured by fluorometric assays. The patient was tested with a small dose of dexamethasone (1 $\mathrm{mg} /$ day, orally), ACTH (0.25 mg, iv) and angiotensin II (5 ng/ $\mathrm{kg} / \mathrm{min}$, div). Adrenal imaging was performed 5 and 7 days after iv administration of $2 \mathrm{mCi}\left[{ }^{131} \mathrm{I}\right]$ adosterol.

Immunohistochemical examinations of steroidogenic enzymes: The resected adrenal glands were fixed in 10\% formalin or 4\% paraformaldehyde solution. After dehydration and paraffin-embedding, the tissues were sectioned and stained with hematoxylin and eosin ( $\mathrm{H}$ and $\mathrm{E}$ ). For immunohistochemical examinations, the sections were stained by the Biotin-Strept avidin amplified method with antibodies to cytochrome P450 (P450scc, P450c21, P450c17, P450c11), 3ß-hydroxysteroid dehydrogenase ( $3 \beta \mathrm{HSD}$ ) as described previously (2-6).

\section{Case Report}

The patient in this study was a 41-year-old woman who was diagnosed with hypertension, which had been treated since 1985. She had a history of toxemia of pregnancy at age 25,28 and 32 years. Her mother had hypertension. The patient was admitted to our hospital for differential diagnosis of hypertension in September 1988. An abdominal computed tomography (CT) image showed round nodules ( $20 \mathrm{~mm}$ in diameter) in the right adrenal area and a nodule ( $15 \mathrm{~mm}$ in diameter) in the left adrenal area (Fig. 1). She had been receiving antihypertensive drugs (spironolactone $30 \mathrm{mg} / \mathrm{day}$, nifedipine $40 \mathrm{mg} / \mathrm{day}$ ). However, she was admitted to our hospital for control of blood

From the Department of Internal Medicine, Kin-ikyo chuo Hospital and *the Department of Pathology, Tohoku University School of Medicine Received for publication May 1, 1996; Accepted for publication September 3, 1996

Reprint requests should be addressed to Dr. Akira Matsuda, the Department of Laboratory Medicine, Kyoto University School of Medicine, Kawahara-cho 54, Shogoin, Sakyo-ku, Kyoto 606 

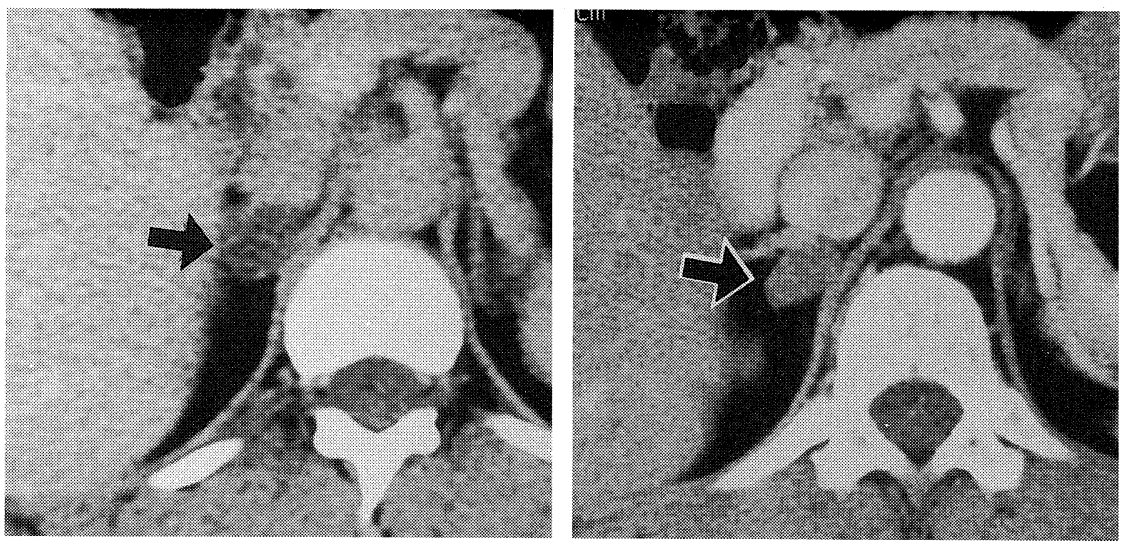

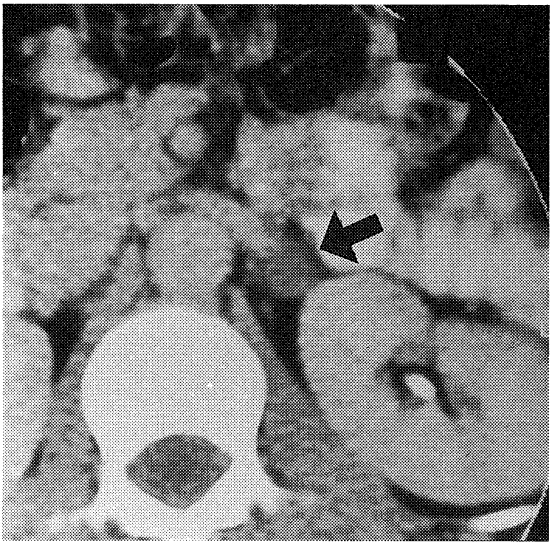

'88.9

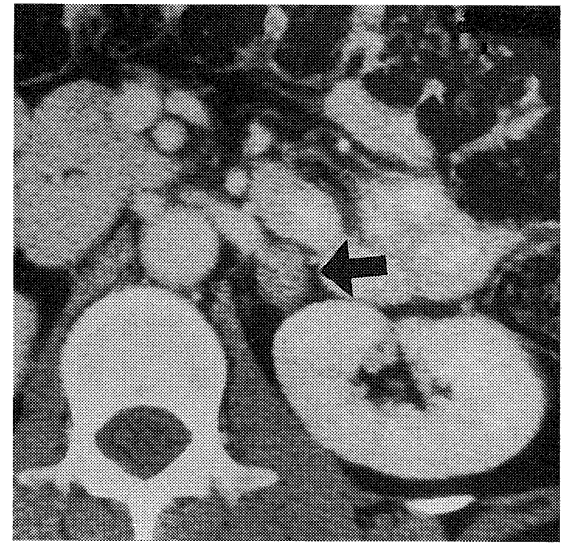

'95.6

Figure 1. CT of the adrenal glands showing bilateral nodules at the first and second admissions.

pressure in January 1995. On admission, she showed muscle weakness, high blood pressure $(160 / 100 \mathrm{mmHg})$ and hypokalemia $(3.0 \mathrm{mEq} / l)$. PAC was elevated $(90.5 \mathrm{ng} / \mathrm{dl}$, normal at supine: $1.0-6.3)$ and PRA was reduced $(0.52 \mathrm{ng} / \mathrm{ml} / \mathrm{h}$, normal: $0.5-2.0)$. The basal urinary $17-\mathrm{OHCS}$ and $17-\mathrm{KS}$ excretion values were $7.0 \mathrm{mg} /$ day (normal: $2-8 \mathrm{mg} /$ day) and 8.7 $\mathrm{mg} /$ day (normal: $3.5-11.6 \mathrm{mg} /$ day), respectively. CT of the adrenal glands showed bilateral nodules, which had not changed in size since the last admission (Fig. 1). MRI of the bilateral adrenal masses showed low-iso in T1 intensity and faint high in $\mathrm{T} 2$ intensity. PAC showed the normal diurnal rhythm, and was suppressed by dexamethasone. When ACTH was administrated, PAC increased significantly from the basal levels of 31.3 $\mathrm{ng} / \mathrm{dl}$ to $130.8 \mathrm{ng} / \mathrm{dl}$ at 60 minutes postinjection. A standing load test showed an increase in PRA, but a decrease in PAC in parallel with serum cortisol levels. When angiotensin II was administrated, PAC did not increase (Fig. 2). These results showed that aldosterone secretion was dependent on ACTH stimulation, which is consistent with adenoma but not hyperplasia. Figure 3 shows the serum concentrations of cortisol (F) and aldosterone in samples obtained from adrenal vein catheterization. The PAC in both adrenal veins greatly exceeded the other vein. The PAC/F ratio did not show laterality in both adrenal veins (Fig. 3).

An adrenal scintigram with $\left[{ }^{131} \mathrm{I}\right]$ adosterol revealed a bilateral uptake of a tracer with a high concentration in both the glands following dexamethasone administration $(2 \mathrm{mg}$ /day for 2 weeks, orally) (Fig. 4). Dexamethasone administration, however, did not improve the serum potassium value or blood pressure.

These results demonstrate that both adrenal glands were hyperfunctional and autonomous. Based on these results, a diagnosis of bilateral APA was made. A partial adrenalectomy was performed at our hospital on July 5, 1995. The operative findings showed 2 nodules ( 21 and $6 \mathrm{~mm}$ in diameter) in the right adrenal side and 1 nodule (16 $\mathrm{mm}$ in diameter) in the left adrenal side. Each nodule including the surrounding adrenal tissue, was resected. There were no complications during the postoperative period. Laboratory findings improved, with 3.8$4.8 \mathrm{mEq} / \mathrm{l}$ in the serum potassium value and $130-150 / 90 \mathrm{mmHg}$ in the level in blood pressure without the administration of any antihypertensive drugs. Macroscopic pathological findings showed a golden-yellow-colored section which was clearly differentiated from the surrounding attached adrenal tissue, and microscopic findings showed a bilateral adrenocortical tumor with a high ratio of compact cells in the right and predominant 


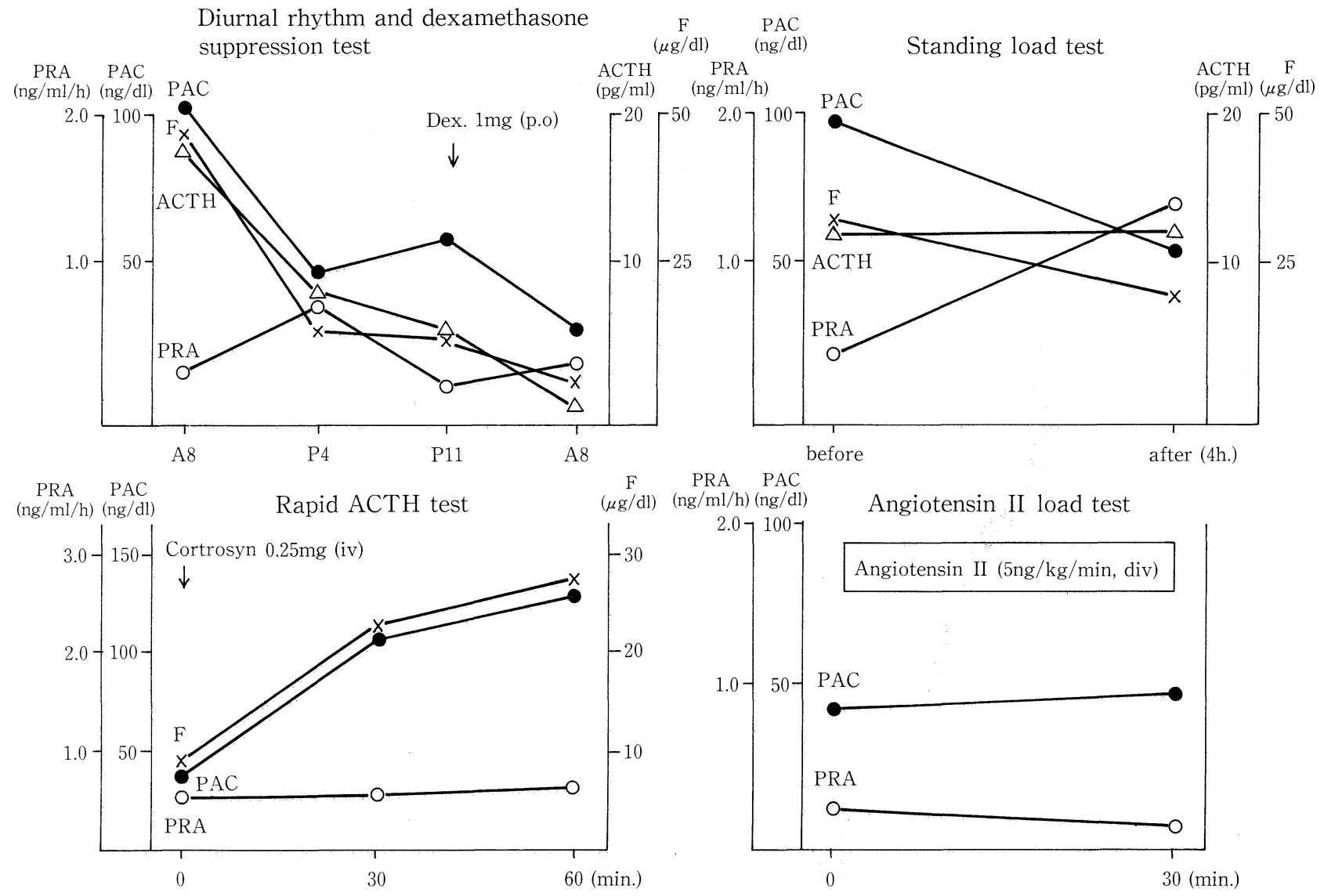

Figure 2. Endocrinological examination. PAC responses in various stimulation tests. PRA, PAC, serum cortisol level (F) and dexamethasone (Dex.).

Th 10

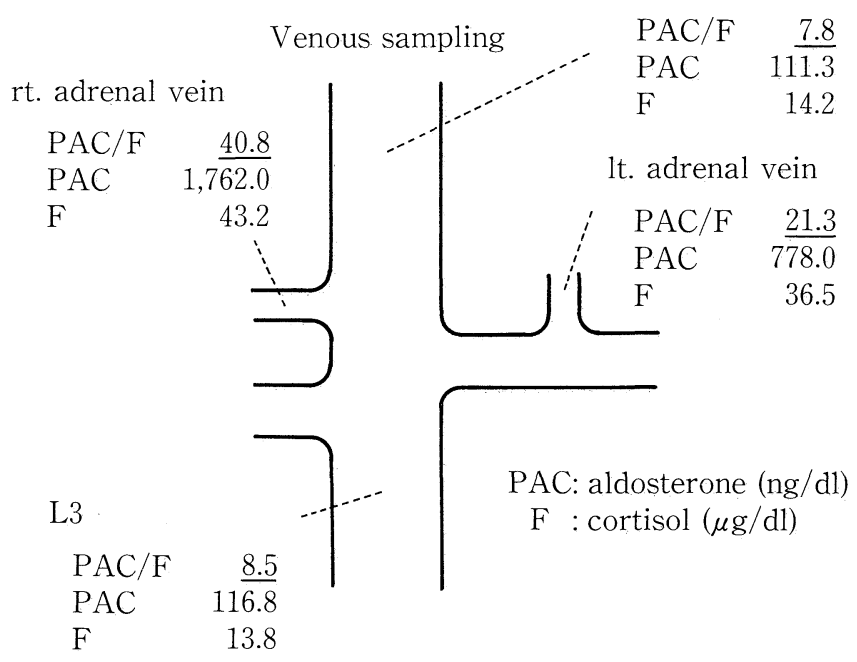

Figure 3. PAC and serum cortisol levels in venous samples selectively collected with a catheter from the adrenal and other related veins, as shown in the scheme.

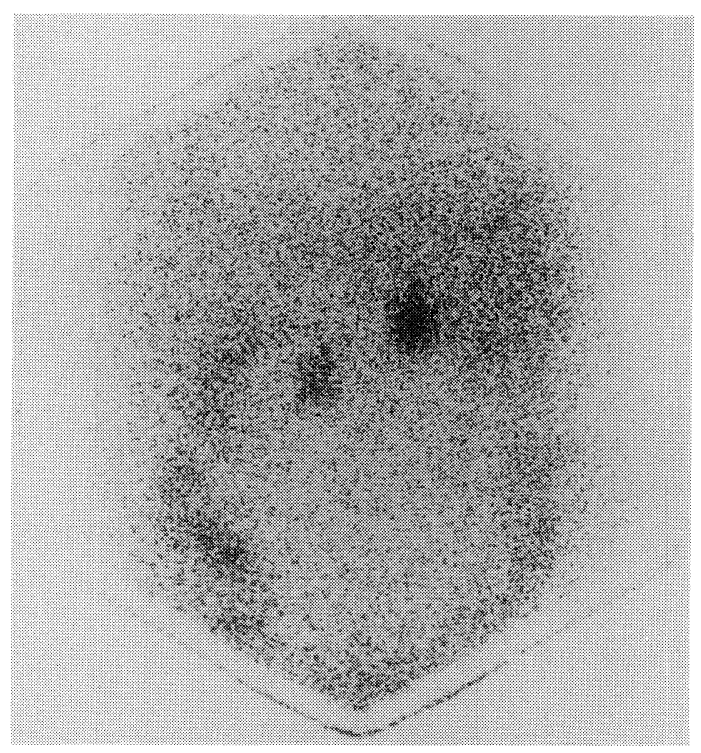

Figure 4. Adrenal scintigraphy using $\left[{ }^{131} I\right]$ adosterol with dexamethasone suppression, showing that both adrenals are functional and autonomous. 
1t. adrenal tumor
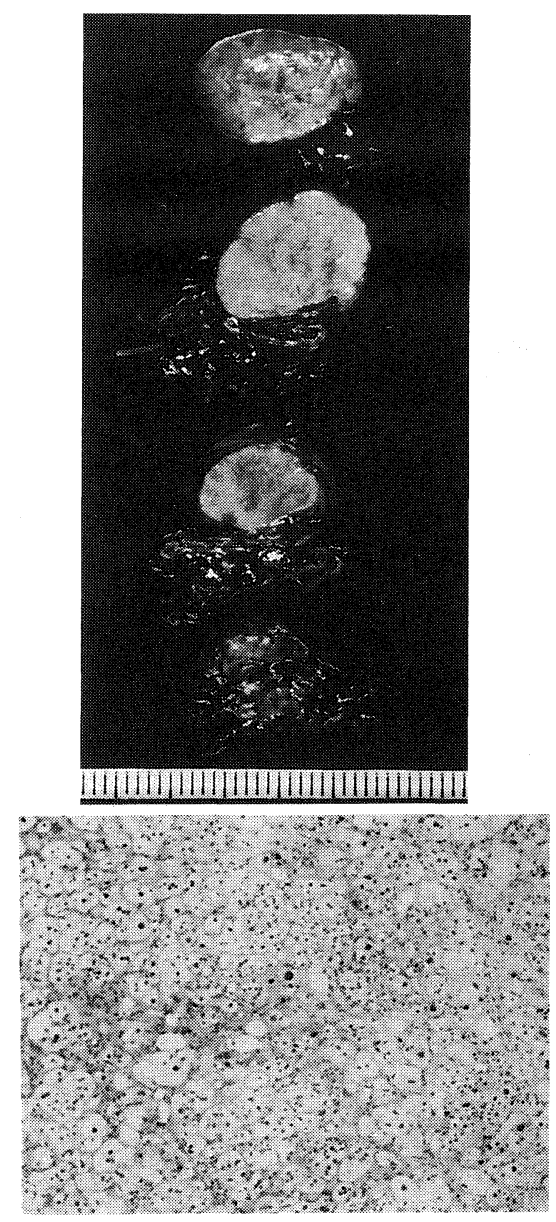

a

attached adrenal

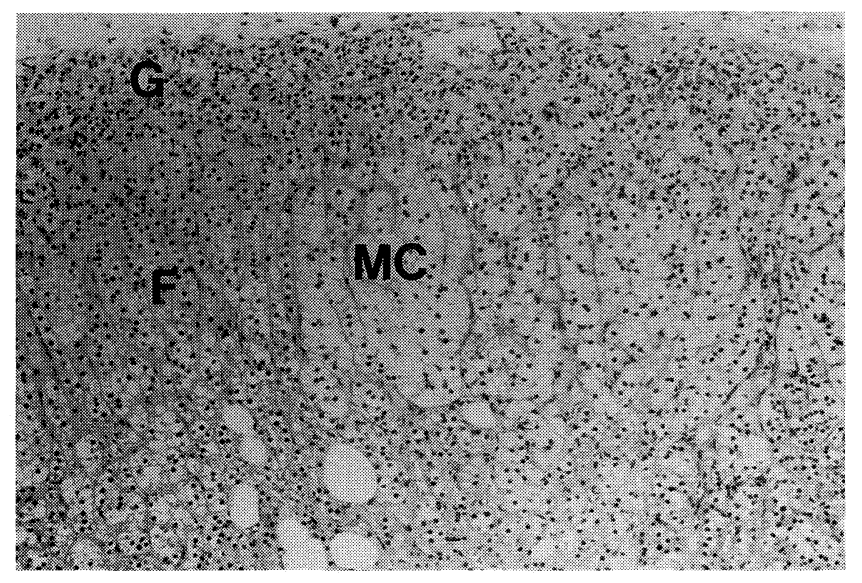

$\mathrm{b}$

clear cells in the left, without any malignant findings (Fig. 5a). Zona glomerulosa of attached adrenal glands showed hyperplasia and some micronodules. Zona fasciculata was not atrophic (Fig. 5b). Immunohistochemical staining demonstrated that the rt. adrenal tumor
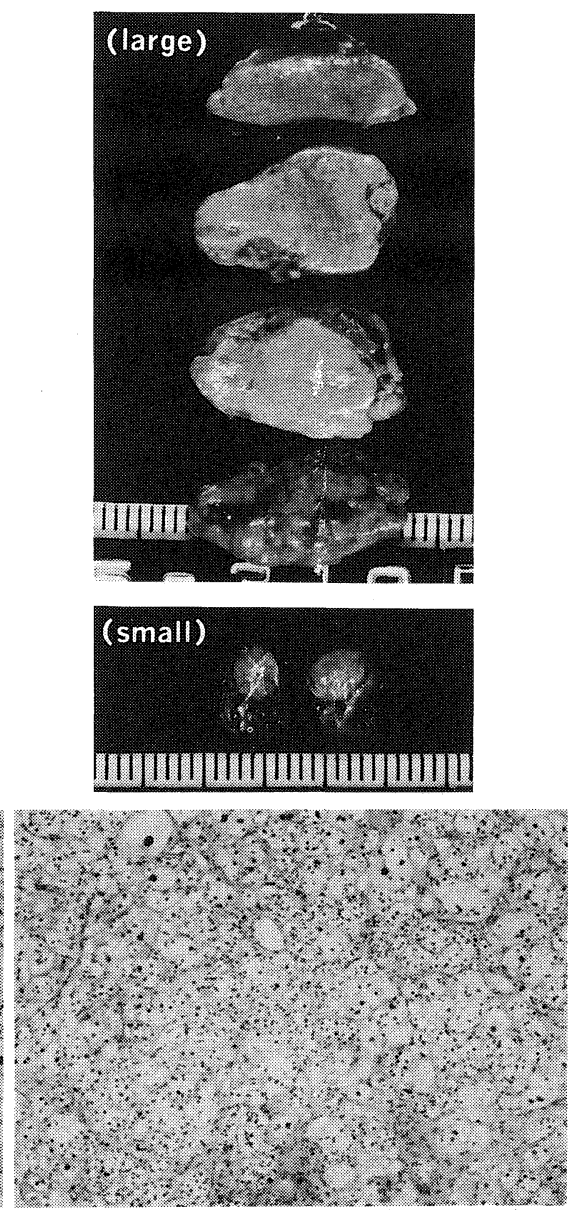

Figure 5. a) Cut sections of both adrenal nodules, showing 2 nodules (21 and $6 \mathrm{~mm}$ in diameter) in the right adrenal and 1 nodule (16 $\mathrm{mm}$ in diameter) in the left adrenal. Light microscopic findings showing a bilateral adrenocortical tumor, which revealed a high ratio of compact cells in the right and predominant clear cells in the left, without malignant findings (HE stain, $\times 50)$. b) Zona glomerulosa of attached adrenal glands showing a hyperplasia and some micronodules and Zona fasciculata is not atrophic by microscopic analysis (HE stain, $\times 100$ ). G: Zona glomerulosa, F: Zona fasciculata, MC: micro nodule.

clear cells of the adrenocortical tumors were positive for $3 \beta$ HSD but not for P450c17, whereas nonneoplastic zona glomerulosa of the attached adrenals were not positive for $3 \beta$ HSD or P450c17 (Fig. 6). Patterns of immunoreactivity of $3 \beta-$ 

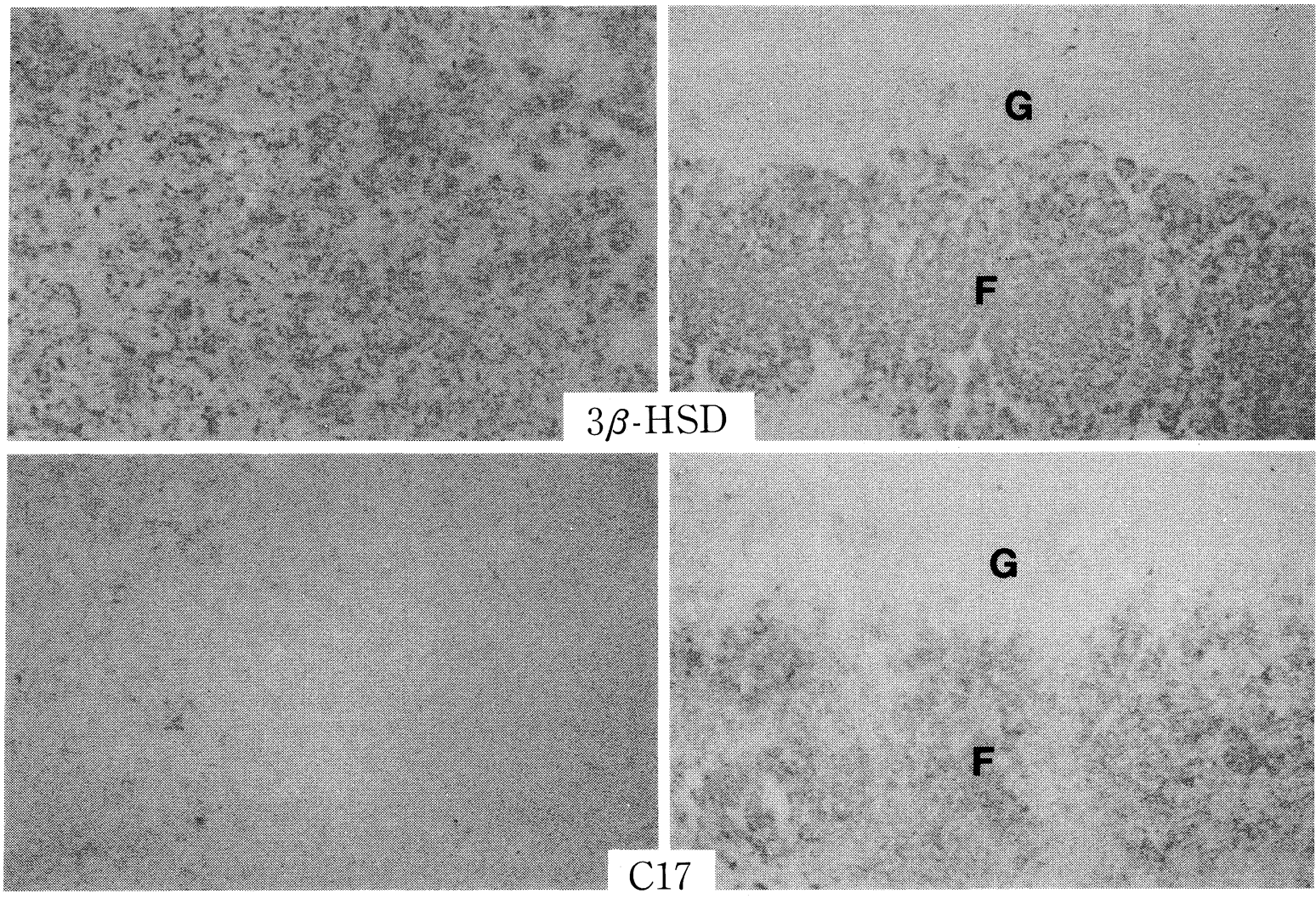

tumor

attached adrenal

Figure 6. Immunohistochemistry of $3 \beta$ HSD and P450c17 in the tumor portions and the zona glomerulosa of the attached adrenals. Immunoreactivities of $3 \beta$ HSD are observed predominantly in the tumor cells, but immunoreactivity

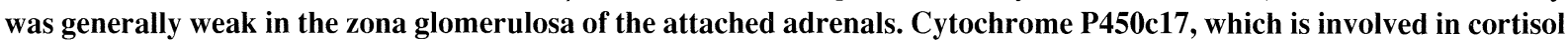
synthesis, is not expressed in either the tumor portions or the zona glomerulosa of the attached adrenals $(\times 100)$. G: Zona glomerulosa, F: Zona fasciculata.

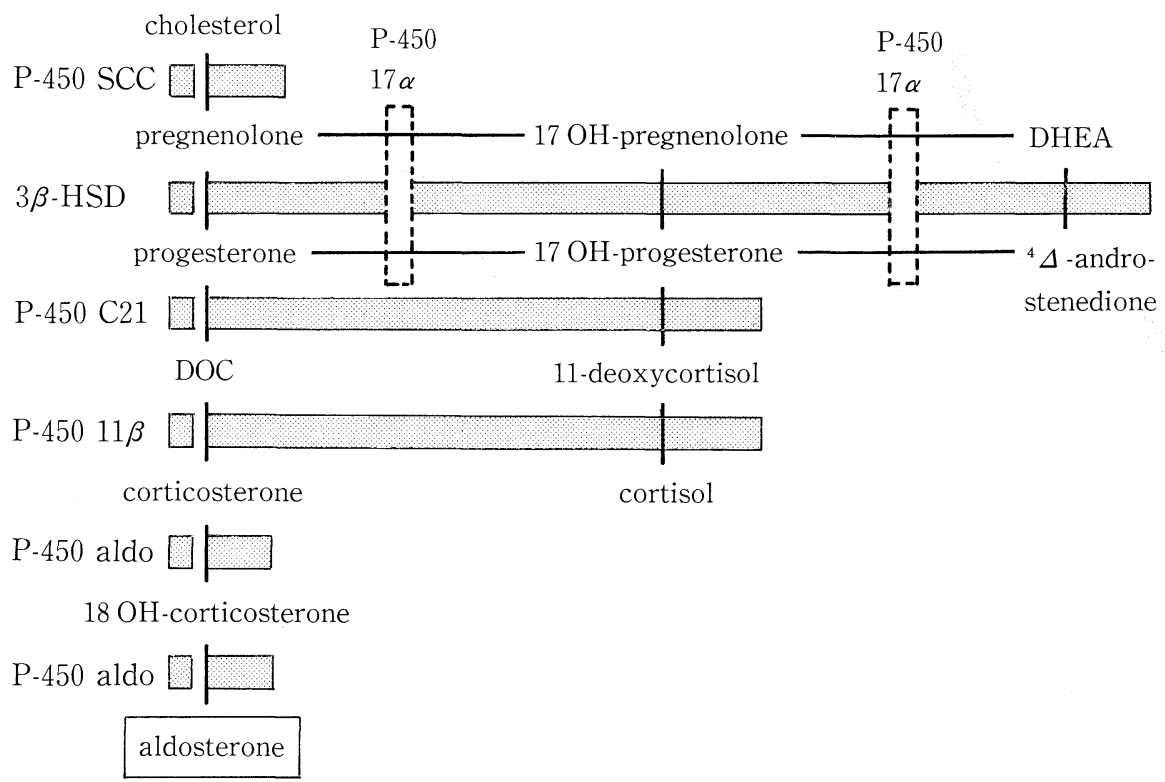

Figure 7. Corticosteroidogenesis pathway in humans.

P450scc: cholesterol side-chain cleavage, P45017 $\alpha$ : 17 $\alpha$-hydroxylase, $3 \beta$ HSD: $3 \beta$ hydroxysteroid dehydrogenase, P450c21: 21-hydroxylase, P45011 $\beta$ : 11 $\beta$-hydroxylase, P450aldo: aldosterone synthetase. 
Table 1. Patterns of Immunohistochemical Staining in This Case

\begin{tabular}{lccccc}
\hline \multicolumn{7}{c}{ P450scc } & $3 \beta$ HSD & P450c21 & P450c11 & P450c17 \\
\hline Tumor & $(+)$ & $(+)$ & $(+)$ & $(+)$ & $(-)$ \\
\hline $\begin{array}{l}\text { Attached adrenal } \\
\text { zona glomerulosa }\end{array}$ & $(-)$ & $(-)$ & $(+)$ & $(-)$ & $(-)$ \\
$\begin{array}{l}\text { micro nodules } \\
\text { zona fasciculata }\end{array}$ & $(+)$ & $(+)$ & $(-)$ & $(-)$ & $(+)$ \\
\hline & & $(+)$ & $(+)$ & $(+)$ & $(+)$ \\
\hline
\end{tabular}

HSD were not consistent in nonneoplastic attached adrenal glands.

In this case, cytochromes P-450 and other enzymes involved in aldosterone synthesis were found predominantly in the tumor portions but not in the glomerulosa of attached adrenals, which histopathologically showed paradoxical hyperplasia and micro nodules. Immunoreactivity of steroidogenic enzymes was normal in the zona fasciculata of attached adrenals (Fig. 7, Table $1)$.

\section{Discussion}

Primary aldosteronism with bilateral adenomas is rare, accounting for only $1.4-2.0 \%$ of primary aldosteronism $(1,2)$. In the present case, results of endocrinological and radiological findings showed APA. Adrenal scintigraphy and venous sampling revealed bilateral adrenal hyperfunction of APA. These results strongly suggested bilateral APA. However, differentiation from nodular hyperplasia (macronodular type) in IHA was difficult (3). Since treatment of these two pathological conditions is different, pathological diagnosis is very important for the determination of a possible clinical cure, together with a preoperative clinical diagnosis (4). However, hyperplasia of attached adrenal tissue is frequently observed in patients with APA, and differentiation between the two pathological conditions is difficult by the typical pathological examination (5).

Recently, Sasano et al developed a technique for the immunohistochemical staining of steroidogenic enzymes in adrenocortical cells $(6,7)$. Using this technique for the present case, we were able to show differential localization of steroidogenic enzymes. In patients with IHA, the adrenal cortex generally shows hyperplasia of the zona glomerulosa with micro nodules (8), which are strongly positive for P450c11 and $3 \beta$ HSD by immunohistochemical staining. In our case, the majority of neoplastic cells were positive for P450scc, P450c21, P450c11 and $3 \beta \mathrm{HSD}$, but not positive in the non-neoplastic attached adrenal glands. This differential localization pattern of steroidogenic enzymes may be a result of ineffective steroidogenesis (aldosterone-producing) with non-neoplastic zona glomerulosa of the attached adrenals showing "paradoxical hyperplasia" and "micro nodules" (5). Therefore, these findings of immunolocalization of steroidogenic enzymes may also be useful for histopathological differential diagnosis of bilateral adrenocortical lesions associated with primary aldosteronism (9).

In conclusion we report a case of bilateral multiple APA, for which differential diagnosis of primary aldosteronism with bilateral adrenal lesions is possible.

\section{References}

1) Conn JW, Knopf RF, Neebit RM. Clinical characteristics of primary aldosteronism from an analysis of 145 cases. Am J Surg 107: 159, 1984.

2) Weinberger MH, Grim CE, Hollifield JW, et al. Primary aldosteronism: Diagnosis, localization, and treatment. Ann Intern Med 90: 386, 1979.

3) Roberts L Jr, Dunnick NR, Thompson WM, et al. Primary aldosteronism due to bilateral nodular hyperplasia: CT demonstration. J Comput Assist Tomogr 9: 1125, 1985.

4) Young WF Jr, Hogan MJ, Klee GG, Grant CS, van Heerden JA. Primary aldosteronism: Diagnosis and treatment. Mayo Clin Proc 65: 96, 1990.

5) Sasano H, Suzuki T. A new histopathological approach to an analysis of human adrenocortical disorders. Pathology and Clinical Medicine 10: 1019, 1992 (in Japanese).

6) Sasano H, Masano JI, Sasano N, Nagura H. Immunolocalization of $3 \beta$ hydroxysteroid dehydrogenase in human adrenal cortex and in its disorders. Endocr Pathol 1: 94, 1990.

7) Sasano H, Masano JI, Sasano N. Immunohistochemical study of cytochrome P-45017 $\alpha$ in human adrenocortical disorders. Hum Pathol 20: $113,1989$.

8) Neville AM, O’Hare MJ. The Human Adrenal Cortex, Pathology and Biology-Integrated Approach. Springer-Verlag, Berlin, Heidelberg, New York, 1982.

9) Sasano H. New approaches in human adrenocortical. Pathology: Assessment of adrenocortical function in surgical specimen of human adrenal glands. Endocr Pathol 3: 4, 1992 (review). 